\title{
Rapid Positive Influence of Cochlear Implantation on the Quality of Life in Adults 70 Years and Older
}

\author{
Heidi Olze Steffen Knopke Stefan Gräbel Agnieszka J. Szczepek \\ Department of Otorhinolaryngology, Head and Neck Surgery, Charité University Hospital, Berlin, Germany
}

\section{Key Words}

Cochlear implantation - Auditory rehabilitation - Quality of life $\cdot$ Tinnitus $\cdot$ Older adults

\begin{abstract}
The deteriorating ability to communicate has a negative impact on quality of life in the aging population. Cochlear implantation is increasingly used to treat hearing impairment and to restore the ability to communicate. Here, in a sample of 79 adults (70 years and older), we explored the effect of cochlear implantation on the health-related quality of life via the Nijmegen Cochlear Implant Questionnaire, auditory performance for speech recognition comparing scores on the Freiburg Monosyllabic Test and Oldenburg Inventory and tinnitus-related distress using the German version of the Tinnitus Questionnaire. We observed that the health-related quality of life and auditory performance increased significantly after cochlear implantation for the study cohort. After implantation, tinnitus-related distress declined significantly for the group. Our results support the concept of cochlear implantation treatment positively influencing the quality of life, restoring the auditory performance in older adults and reducing stress related to tinnitus. Importantly, positive effects were seen as early as 6 months after cochlear implantation, corroborating the rationale for cochlear implantation in adults 70 years and older.

(C) 2016 The Author(s)

Published by S. Karger AG, Basel
\end{abstract}

\section{Introduction}

Hearing loss is a prevalent health condition affecting older adults in a particular age-related way. Elderly people who are hard of hearing have problems with communication and report a decrease in the quality of life and physical and cognitive activities [Bainbridge and Wallhagen, 2014]. Multiple studies have shown that hearing loss not only incapacitates the ability to communicate, but also that it is positively associated with depression, somatization disorder, and loneliness in older adults [Ciorba et al., 2012; Sung et al., 2015]. In addition, hearing loss was found to be associated with an increased risk of developing dementia [Fortunato et al., 2016; Lin et al., 2011; Peracino, 2014]. Although the major goal of cochlear implantation is auditory rehabilitation, other positive consequences of cochlear implantation have been noted, namely improvement in the general and health-related quality of life (HRQoL) [Fuller et al., 2013; Karinen et al., 2001; Rumeau et al., 2015]. The issue of healthy aging becomes increasingly important as the life expectancy in industrialized countries is on the rise. Moreover, the number of adults who are 70 years and older referred for cochlear implantation to our clinic has steadily increased in the last decade (fig. 1). Recently, a major literature review identified the need for more research on changes in psychosocial and functional status following cochlear implantation of older adults [Clark et al., 2012]. The gap in

\section{KARGER}

E-Mail karger@karger.com www.karger.com/aud

\section{(C) 2016 The Author(s)}

Published by S. Karger AG, Basel 1420-3030/16/0217-0043\$39.50/0

This article is licensed under the Creative Commons AttributionNonCommercial-NoDerivatives 4.0 International License (CC BYNC-ND) (http://www.karger.com/Services/OpenAccessLicense) Usage and distribution for commercial purposes as well as any distribution of modified material requires written permission.
Prof. Dr. med. Heidi Olze

Department of Otorhinolaryngology, Head and Neck Surgery

CCM and CVK, Charité University Hospital

Augustenburgerplatz 1, DE-13353 Berlin (Germany)

E-Mail Heidi.Olze@ charite.de 
Fig. 1. Steady rise in the number of patients who are 70 years and older referred in the last decade for cochlear implantation to our unit at the Charité University Hospital in Berlin.

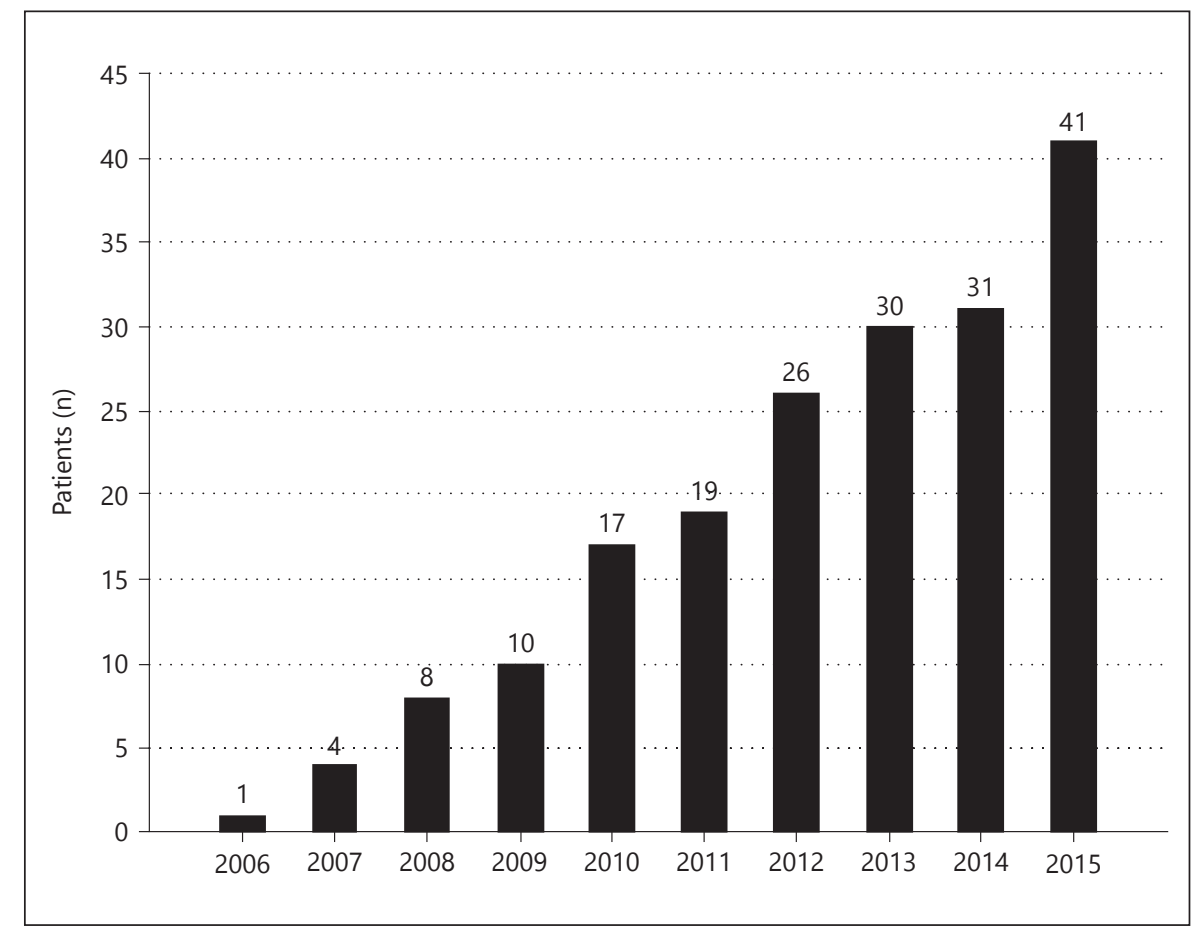

knowledge is gradually being filled with the medical-evidence data generated from research by our group and that of others [Cloutier et al., 2014; Contrera et al., 2016; Knopke et al., 2016; Olze et al., 2012]; however, performing studies with larger samples, measuring more parameters such as cognition [Mosnier et al., 2015] and using a prospective longitudinal study design, is essential for further justification of cochlear implantation treatment in older adults.

In our earlier retrospective study in a small group of 20 patients over the age of 70 years, we have shown that there was significant improvement in the HRQoL, a reduction in tinnitus-related distress and a decrease in perceived stress following cochlear implantation [Olze et al., 2012]. Recently, we reported similar results for $17 \mathrm{im}$ planted patients who were 80 years or older [Knopke et al., 2016]. In our present work, we applied a prospective, longitudinal, repeated-measures study design to investigate the influence of cochlear implantation on HRQoL, auditory performance, and the degree of tinnitus-related distress in a larger study cohort of 79 adult cochlear implant (CI) recipients aged 70 and over. The data analysis presented represents their outcome data at 6 months after cochlear implantation compared to their preimplantation status.

\section{Materials and Methods}

This prospective study was approved by the local Charité University Hospital Ethics Committee. All investigations have been conducted according to the principles expressed in the Declaration of Helsinki.

\section{Patients' Characteristics}

Seventy-nine patients (44 women and 35 men) who were aged 70 years or over at the time of implantation (mean age 75.4, range 70-88 years) participated in the study upon signing consent. All recruited patients were consecutively admitted to be implanted because of uni- or bilateral sensorineural hearing impairment (17 and 62 patients, respectively).

\section{Outcome Measures}

All questionnaires used in this study have been described previously in detail [Knopke et al., 2016; Olze et al., 2012]. Briefly, the Nijmegen Cochlear Implant Questionnaire (NCIQ) was used to measure changes in the HRQoL, the Oldenburg Inventory was used to assess the subjective auditory changes and the German version of the Tinnitus Questionnaire was used to assess the tinnitusrelated distress. The Freiburg Monosyllabic Word Test was used to determine the preoperative speech recognition scores in quiet at $65 \mathrm{~dB}$ SPL with an optimized hearing aid that was worn by all but one subject prior to implantation. This test was performed in quiet at $65 \mathrm{~dB}$ SPL after implantation.

\section{Statistical Analyses}

The analyses were performed with SPSS version 23. The MannWhitney $\mathrm{U}$ test was applied to determine differences between the 


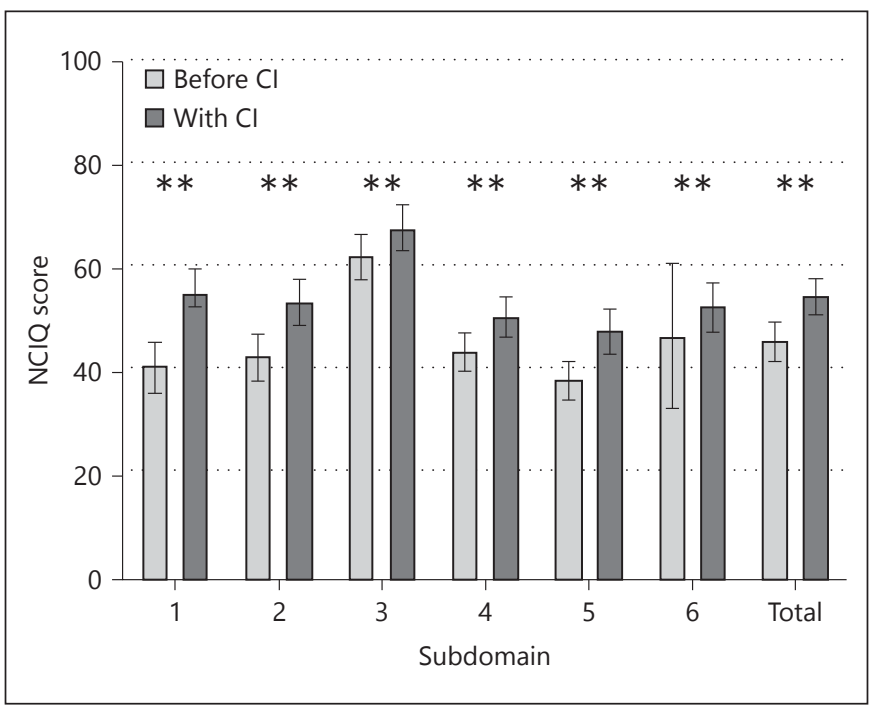

Fig. 2. Improvement in the HRQoL in patients 70 years and older following cochlear implantation measured on the NCIQ. Subdomains: 1 = 'basic sound perception', 2 = 'advanced sound perception', 3 = 'speech production', 4 = 'self-esteem', 5 = 'activity', and $6=$ 'social interactions'. ${ }^{* *} \mathrm{p}<0.01$.

matched pair data sets; some comparisons between pre- and postoperative results were performed using the Wilcoxon test. The significance levels were set at 0.05 or less.

\section{Results}

The HRQoL was assessed before and 6 months after cochlear implantation with the NCIQ (fig. 2). Statistically significant improvement $(\mathrm{p}<0.01)$ was noted for all measured subdomains (1: basic sound perception, 2: advanced sound perception, 3: speech production, 4: selfesteem, 5: activity, and 6: social interactions), as well as in the total NCIQ score.

Standardly performed clinical monitoring with the Oldenburg Test Inventory, before and after cochlear implantation, confirmed the appropriate development of auditory rehabilitation reflected by significant improvement of hearing in quiet and noise, as well as for sound localization (fig. 3). Freiburg Monosyllabic Word Test results also indicated a significant improvement in speech recognition comparing data obtained before implantation (average 6.73; SD 11.84) to that after implantation (average 33.16; SD 27.56; $\mathrm{p}=0.000$ ).

Lastly, tinnitus-related distress was measured in all patients before and after implantation. In our sample, 70\% (55 of 79) tested positive for tinnitus at the preimplanta-

Cochlear Implantation and Quality of Life for 70 Years Plus

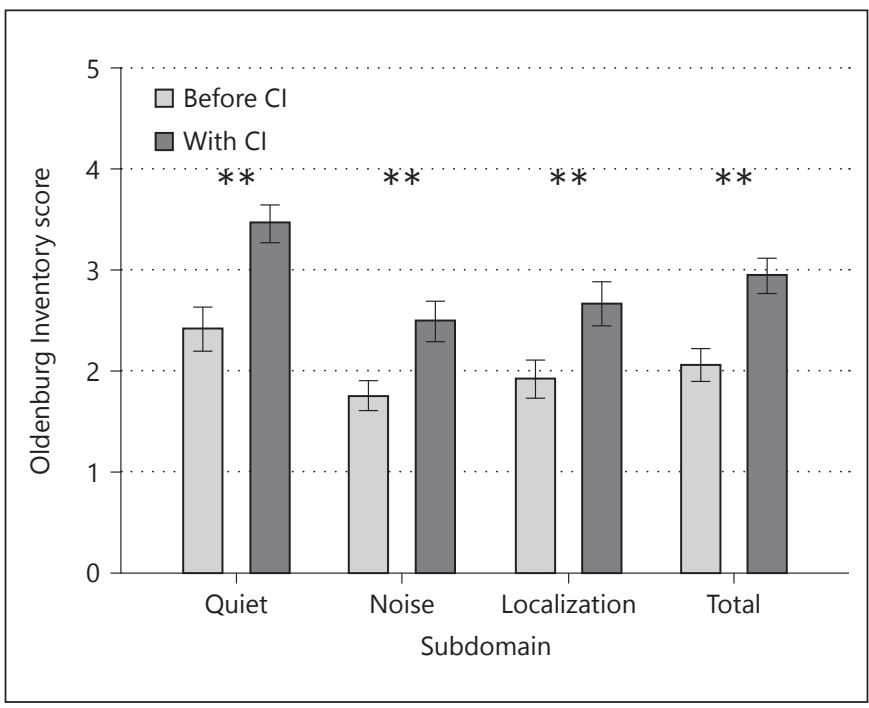

Fig. 3. Results of the Oldenburg Inventory showing positive outcomes of CI-dependent auditory rehabilitation in patients 70 years and older. ${ }^{* *} \mathrm{p}<0.01$.

tion interval. In the tinnitus-positive group, 32 subjects reported improvement, 8 no change, and 15 worsening of tinnitus-related distress. In the latter group, only 3 patients reported worsening by a clinically relevant score of 15 points or more. The total Tinnitus Questionnaire score decreased significantly after cochlear implantation treatment (fig. 4), as did the majority of specific subscale scores (emotional distress, cognitive distress, intrusiveness, auditory perceptional difficulties, somatic complaints); however, no significant change was noted for 'sleep disturbances'.

\section{Discussion}

The results of our study demonstrate that adult CI recipients who are 70 years and older at implantation benefit from the cochlear implantation after 6 months of experience. Importantly, not only a positive outcome of auditory rehabilitation was seen, but also significant improvement of the quality of life and reduction of tinnitusinduced distress. The main goal of cochlear implantation is an improvement of hearing and speech understanding; however, restoring the ability to hear enhances the quality of life and reduces tinnitus-related distress. Data regarding patients who are 70 years or older are scarce but, due to the aging of our society, it is precisely this growing 


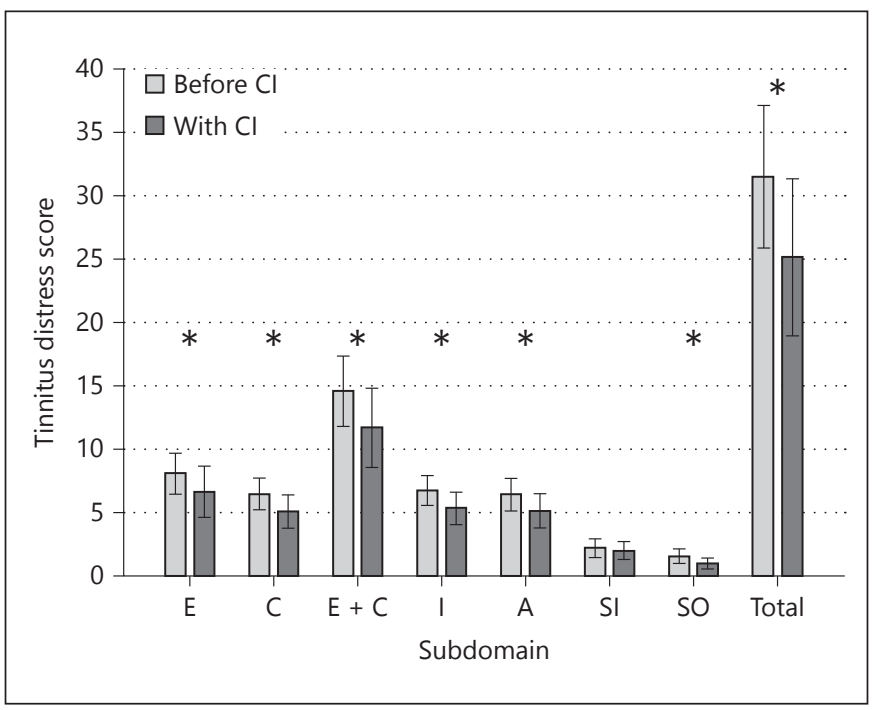

Fig. 4. Tinnitus-related distress decreases in patients 70 years and older following cochlear implantation measured on the Tinnitus Questionnaire. $\mathrm{E}=$ 'Emotional distress'; $\mathrm{C}=$ 'cognitive distress'; $\mathrm{E}+\mathrm{C}=$ sum of 'emotional and cognitive distress'; $\mathrm{I}=$ 'intrusiveness'; $\mathrm{A}=$ = 'auditory perceptional difficulties'; $\mathrm{Sl}=$ 'sleep disturbances'; $\mathrm{SO}=$ 'somatic complaints'. ${ }^{*} \mathrm{p}<0.05$.

population that is now the focus of contemporary medical and psychosocial studies.

In our earlier comparative study of patients under and over 70 years of age [Olze et al., 2012], we demonstrated that the HRQoL increased in all patients following cochlear implantation but to a greater degree in the older than in the younger group. We attributed this difference to the fact that older patients had a higher degree of HRQoL impairment at the onset of the study. The main pitfalls of that study were its retrospective design and the inclusion of only long-term measures after surgery (21 months), likely causing memory recall bias. In our present study, we measured the HRQoL after 6 months of experience with the $\mathrm{CI}$ and, for the first time, demonstrated positive outcomes for psychological (self-esteem) and social domains.

Researchers have also reported an increase in the quality of life following cochlear implantation (measured with the Glasgow Benefit Inventory) in a group of 79 CI patients of whom 25 were 70 years or older [Vermeire et al., 2005]. In addition, the authors noted a positive correlation between the HRQoL and the age of patients. Nevertheless, their sample size included a relatively small subgroup of $>70$-year-olds. A recent retrospective, postoperative study report on CI patients who were 80 years or older also observed a noticeable improvement in HRQoL [Cloutier et al., 2014]. However, the mean time between the surgery and reporting the quality of life was 3.8 years, with a likely bias of memory recall.

The improvement in HRQoL seen after cochlear implantation is associated with the improvement of speech understanding. The consequence of a permanent hearing impairment is a combination of cognitive deficiency and social isolation. Furthermore, a worsening of cognitive performance and syndromes of dementia, as well as a reduction of physical abilities, are also possible consequences [Lin, 2011]. For this reason, appropriate hearing rehabilitation including cochlear implantation, where indicated by the degree of hearing loss is required regardless of the patient's age, which is not a contraindication for cochlear implantation [Haensel et al., 2005]. As reported by researchers, the quality of life was significantly improved following cochlear implantation in a group of patients between 50 and 80 years of age [Francis et al., 2002]. In addition, a significant correlation between the auditory gain and the HRQoL has been found. Taken together, our prospective study corroborates other studies and adds statistical weight due to the relatively large sample size and the nature of the intrasubject study design, bringing new information about the early, positive effects of implantation in adults who are 70 years and older.

Conforming to our earlier findings in the retrospective study on a smaller sample size, we have observed significant improvement in auditory performance of our patients. However in the present study, we determined that the auditory improvement occurs already within 6 months after implantation, with improved performance on speech recognition in quiet and in noise and for sound localization. In agreement with our observations, the report of a retrospective study with patients 79 years or older also demonstrated a significant improvement in auditory performance [Eshraghi et al., 2009]. Similarly in another retrospective work, a significant increase in speech recognition in older CI recipients was observed [Cloutier et al., 2014].

Patients with auditory deficits have a well-known high prevalence of tinnitus. In a systematic review where 1,104 CI candidates were analyzed, Baguley and Atlas [2007] report a prevalence of tinnitus between 67 and 100\% with an average of $80 \%$. Additionally, unilaterally CI-implanted patients reported a decrease in tinnitus ranging from 36 to $83 \%$ following implantation. Similarly, we have previously demonstrated that cochlear implantation of patients with a severe hearing impairment leads to a significant reduction of tinnitus distress [Olze et al., 2011, 
2012]. Our present study confirmed these findings, as we determined a significant reduction in tinnitus-related distress. Our data agree with the general tinnitus prevalence found in CI users of all ages [Baguley and Atlas, 2007], suggesting that the prevalence of tinnitus in hardof-hearing individuals is age independent.

\section{Conclusions}

In summary, we report on the positive result of cochlear implantation in older adults ( $>70$ years). The outcomes measured as early as 6 months after surgery included not only auditory performance, but also HRQoL and tinnitus-related distress. We conclude that cochlear implantation is an important means of auditory rehabilitation for older adults, that significant benefits of implantation are seen already 6 months following surgery and that the benefits of a CI extend beyond the auditory system and positively affect social and cognitive domains, suggesting that cochlear implantation of hearing-impaired adults $>70$ years of age contributes to healthy aging.

\section{Disclosure Statement}

The authors report no conflict of interest.

\section{References}

Baguley DM, Atlas MD: Cochlear implants and tinnitus. Prog Brain Res 2007;166:347-355.

Bainbridge KE, Wallhagen MI: Hearing loss in an aging American population: extent, impact, and management. Annu Rev Public Health 2014;35:139-152.

Ciorba A, Bianchini C, Pelucchi S, Pastore A: The impact of hearing loss on the quality of life of elderly adults. Clin Interv Aging 2012;7:159163.

Clark JH, Yeagle J, Arbaje AI, Lin FR, Niparko JK, Francis HW: Cochlear implant rehabilitation in older adults: literature review and proposal of a conceptual framework. J Am Geriatr Soc 2012;60:1936-1945.

Cloutier F, Bussieres R, Ferron P, Cote M: OCTO 'Outcomes of cochlear implant for the octogenarians: audiologic and quality-of-life'. Otol Neurotol 2014;35:22-28.

Contrera KJ, Betz J, Li L, Blake CR, Sung YK, Choi JS, Lin FR: Quality of life after intervention with a cochlear implant or hearing aid. Laryngoscope 2016, Epub ahead of print.

Eshraghi AA, Rodriguez M, Balkany TJ, Telischi FF, Angeli S, Hodges AV, Adil E: Cochlear implant surgery in patients more than seventy-nine years old. Laryngoscope 2009; 119: 1180-1183.

Fortunato S, Forli F, Guglielmi V, De Corso E, Paludetti G, Berrettini S, Fetoni AR: A review of new insights on the association between hearing loss and cognitive decline in ageing. Acta Otorhinolaryngol Ital 2016;36:155-166.
Francis HW, Chee N, Yeagle J, Cheng A, Niparko JK: Impact of cochlear implants on the functional health status of older adults. Laryngoscope 2002;112:1482-1488.

Fuller C, Mallinckrodt L, Maat B, Baskent D, Free R: Music and quality of life in early-deafened late-implanted adult cochlear implant users. Otol Neurotol 2013;34:1041-1047.

Haensel J, Ilgner J, Chen YS, Thuermer C, Westhofen M: Speech perception in elderly patients following cochlear implantation. Acta Otolaryngol 2005;125:1272-1276.

Karinen PJ, Sorri MJ, Valimaa TT, Huttunen KH, Lopponen HJ: Cochlear implant patients and quality of life. Scand Audiol Suppl 2001;52: 48-50.

Knopke S, Gräbel S, Förster-Ruhrmann U, Mazurek B, Szczepek AJ, Olze H: Impact of cochlear implantation on quality of life and mental comorbidity in patients aged 80 years. Laryngoscope 2016, Epub ahead of print.

Lin FR: Hearing loss and cognition among older adults in the United States. J Gerontol A Biol Sci Med Sci 2011;66:1131-1136.

Lin FR, Metter E, O’Brien RJ, Resnick SM, Zonderman AB, Ferrucci L: Hearing loss and incident dementia. Arch Neurol 2011;68:214220.
Mosnier I, Bebear JP, Marx M, Fraysse B, Truy E, Lina-Granade G, Mondain M, Sterkers-Artieres F, Bordure P, Robier A, Godey B, Meyer B, Frachet B, Poncet-Wallet C, Bouccara D, Sterkers O: Improvement of cognitive function after cochlear implantation in elderly patients. JAMA Otolaryngol Head Neck Surg 2015;141:442-450.

Olze H, Gräbel S, Förster U, Zirke N, Huhnd LE, Haupt H, Mazurek B: Elderly patients benefit from cochlear implantation regarding auditory rehabilitation, quality of life, tinnitus, and stress. Laryngoscope 2012;122:196-203.

Olze H, Szczepek AJ, Haupt H, Forster U, Zirke N, Grabel S, Mazurek B: Cochlear implantation has a positive influence on quality of life, tinnitus, and psychological comorbidity. Laryngoscope 2011;121:2220-2227.

Peracino A: Hearing loss and dementia in the aging population. Audiol Neurootol 2014;19 (suppl 1):6-9.

Rumeau C, Frere J, Montaut-Verient B, Lion A, Gauchard G, Parietti-Winkler C: Quality of life and audiologic performance through the ability to phone of cochlear implant users. Eur Arch Otorhinolaryngol 2015;272:3685-3692.

Sung YK, Li L, Blake C, Betz J, Lin FR: Association of hearing loss and loneliness in older adults. J Aging Health 2015, Epub ahead of print.

Vermeire K, Brokx JPL, Wuyts FL, Cochet E, Hofkens A, Van de Heyning PH: Quality-oflife benefit from cochlear implantation in the elderly. Otol Neurotol 2005;26:188-195. 